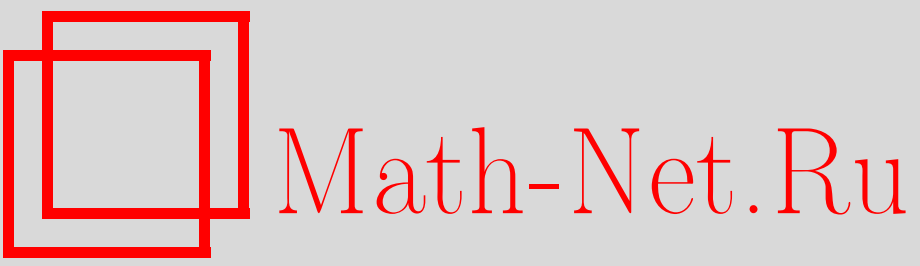

А. С. Лыскова, Полиномиальные решения некоторого класса обыкновенных дифференциальных уравнений, УМН, 1996, том 51, выпуск 3, 207-208

DOI: https://doi.org/10.4213/rm987

Использование Общероссийского математического портала Math-Net.Ru подразумевает, что вы прочитали и согласны с пользовательским соглашением

http://www.mathnet.ru/rus/agreement

Параметры загрузки:

IP : 54.157 .27 .8

26 апреля 2023 г., 14:26:49 


\title{
ПОЛИНОМИАЛЬНЫЕ РЕШЕНИЯ НЕКОТОРОГО КЛАССА ОБЫКНОВЕННЫХ ДИФФЕРЕНЦИАЛЬНЫХ УРАВНЕНИЙ
}

\author{
А. С. ЛысковА
}

В настоящей работе рассматривается задача на собственные значения для дифференциального уравнения следующего типа:

$$
p_{n} y^{(n)}+p_{n-1} y^{(n-1)}+\cdots+p_{1} y^{\prime}+\lambda y=0,
$$

где $p_{i}(x)$ - многочлен степени не выше $i$, и исследуется вопрос об ортогональности собственных функций с весом $\rho(x)$ на некотором интервале $(a, b)$.

Известно, что для случая $n=2$ решения уравнения (0) являются классическими ортогональными многочленами.

Решения уравнения (0) обладают замечательным свойством: производные любого порядка от этих решений также удовлетворяют уравнению типа (0). Используя метод, предложенный в работе [1], можно доказать аналогичные теоремы для уравнения (0) произвольного порядка.

Теорема 1. Если $y=y(x)-$ решения уравнения $(0)$, то $v_{k}(x)=y^{(k)}(x)-$ решение уравнения $(\mathrm{k})$ такого же типа:

$$
\sum_{i=1}^{n} p_{i}^{k} v_{k}^{(i)}+\lambda_{k} v_{k}=0, \quad k=1,2, \ldots
$$

При этом коэффициенты $p_{i}^{k}$ и $p_{i}$ связань следующими соотношениями:

$$
\begin{aligned}
p_{j}^{k} & =p_{j}+C_{k}^{1} p_{j+1}^{\prime}+C_{k}^{2} p_{j+2}^{\prime \prime}+\cdots+C_{k}^{n-j} p_{n}^{(n-j)}, \\
\lambda_{k} & =\lambda+C_{k}^{1} p_{1}^{\prime}+C_{k}^{2} p_{2}^{\prime \prime}+\cdots+C_{k}^{(n)} p_{n}^{(n)}
\end{aligned}
$$

где $C_{k}^{j}=0$ при $j>k, j=1,2, \ldots, n$.

Справедливо и обратное утверждение.

Теорема $1^{\prime}$. Любое решение уравнения $(\mathrm{k})$ при $\lambda_{k} \neq 0, k=1,2, \ldots$, можно представить в виде $v_{k}(x)=y^{(k)}(x)$, где $y(x)$ - некоторое решение уравнения $(0)$.

Рассмотренное свойство позволяет построить семейство частных решений уравнения $(0)$, соответствующих определенным значениям $\lambda$.

Теорема 2. Существуют частные решения уравнения (0), являющиеся полиномами степени $k$ и отвечающие собственным значениям $\lambda_{k}$ :

$$
\lambda_{k}=-C_{k}^{1} p_{1}^{\prime}-C_{k}^{2} p_{2}^{\prime \prime}-\ldots-C_{k}^{(n)} p_{n}^{(n)},
$$

əде $C_{k}^{j}=0$ nрu $j>k$.

Исследуем вопрос об ортогональности многочленов и их производных с некоторой плотностью $\rho(x)$ на интервале $(a, b)$.

Известно, что всякое самосопряженное дифференциальное выражение с действительными коэффициентами является суммой дифференциальных выражений четного порядка $l_{2 \nu}(y)=$ $\left(q(x) y^{(\nu)}\right)^{(\nu)}, \nu=1,2, \ldots$,

$$
l(y)=\sum_{i=0}^{\mu}\left(q_{2 i}(x) y^{(i)}(x)\right)^{(i)},
$$

где $q_{2 \mu}(x), q_{2(\mu-1)}(x), \ldots, q_{0}(x)$ - вещественные функции. 
Умножим уравнения $(0)$ и $(\mathrm{k})$ на такие функции $\rho(x)$ и $\rho_{k}(x)$, чтобы записать их в самосопряженном виде:

$$
\begin{gathered}
\sum_{i=1}^{n} \rho p_{i} y^{(i)}+\lambda \rho y=\sum_{i=1}^{\mu}\left(q_{2 i} y^{(i)}\right)^{(i)}+\lambda \rho y \\
\sum_{i=1}^{n} \rho_{k} p_{i}^{k} v_{k}^{(i)}+\mu_{k} \rho_{k} v_{k}=\sum_{i=1}^{\mu}\left(q_{2 i}^{k} v_{k}^{(i)}\right)^{(i)}+\mu_{k} \rho_{k} v_{k} .
\end{gathered}
$$

Из равенств (5) и (6) следует, что $n=2 \mu$ и функции $\rho(x), \rho_{k}(x)$ удовлетворяют дифференциальным уравнениям:

$$
\begin{aligned}
\rho p_{n-1} & =C_{\mu}^{1}\left(\rho p_{n}\right)^{\prime}, \\
\rho_{k} p_{n-1}^{k} & =C_{\mu}^{1}\left(\rho_{k} p_{n}\right)^{\prime},
\end{aligned}
$$

откуда получаем, что

$$
\frac{\rho^{\prime}}{\rho}+\frac{k}{\mu} \frac{p_{n}^{\prime}}{p_{n}}=\frac{\rho_{k}^{\prime}}{\rho_{k}}
$$

и, следовательно, $\rho_{k}=\rho\left(p_{n}\right)^{2 k / n}$.

Из уравнений $(7)$ и $(8)$ можно найти плотности $\rho(x)$ и $\rho_{k}(x)$. Для любых многочленов $u, v$ справедлива формула Лагранжа (см. [3])

$$
\int_{a}^{b}(l u) v d x=\int_{a}^{b} u(l v) d x+P(\eta, \xi)
$$

где $P(\eta, \xi)$ - некоторая билинейная форма относительно

$$
\eta=\left(u_{a}, u_{a}^{\prime}, \ldots, u_{a}^{(n-1)}, u_{b}, u_{b}^{\prime}, \ldots, u_{b}^{(n-1)}\right), \quad \xi=\left(v_{a}, v_{a}^{\prime}, \ldots, v_{a}^{(n-1)}, v_{b}, v_{b}^{\prime}, \ldots, v_{b}^{(n-1)}\right) .
$$

Если $P(\eta, \xi)=0$ (например, если $\left.x^{k} \rho(x)\right|_{x=a}=\left.x^{k} \rho(x)\right|_{x=b}=0 \forall k \in \mathbb{N}$ ), то многочленш $u(x)$, $v(x)$ ортогональны на интервале $(a, b)$ с плотностью $\rho(x)$. То есть справедлива теорема.

Теорема 3. Если выполнены граничные условия $P(\eta, \xi)=0$, то решения $y(x)$ уравнения (0) и их производные $v_{k}(x)=y^{(k)}(x)$ ортогональны на интервале $(a, b)$ с плотностями $\rho(x)$ и $\rho_{k}(x)=\rho\left(p_{n}\right)^{2 k / n}$, соответственно.

\section{СПИСОК ЛИТЕРАТУРЫ}

[1] Никифоров А.Ф., Уваров В. Б. Основы теории специальных функций. М.: Наука, 1974. [2] Суетин П.К. Ортогональные многочлены по двум переменным. М.: Наука, 1988. [3] Костюченко А. Г., Саргсян И. С. Распределение собственных значений. М.: Наука, 1979. 